\title{
Un banco de leche para Chile
}

\author{
P. Mena
}

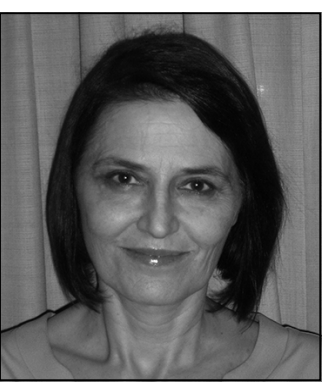

La donación de leche, a través de nodrizas, $\mathrm{y}$ de bancos de leche en el siglo XX, tuvo un rol en la atención del recién nacido y lactante. En Chile, los bancos de leche humana existieron en algunas maternidades y hospitales pediátricos hasta 1988 y sólo una maternidad pública lo mantuvo más años. En algunas maternidades no se usó leche donada debido a las ventajas nutricionales de la leche de su propia madre, que contiene cantidades significativamente mayores de proteínas, con lo que se produce un mejor crecimiento, que con la leche de banco de madre de término. Por este motivo se generalizó el uso de lactarios, en que una madre se extrae leche, es conservada y administrada para su propio hijo.

El cierre de los bancos de leche fue determinado por el potencial riesgo de transmisión de virus VIH. Posteriormente, al demostrarse la inactivación del virus por pasteurización, los bancos de leche se reiniciaron o continuaron en distintas partes del mundo, especialmente en Brasil, donde los recién nacidos y lactantes hospitalizados reciben leche humana (LH) donada pasteurizada ${ }^{1}$. En los últimos 20 años se han establecido una gran cantidad de bancos de leche, en todos los países de Europa, en Australia, Nueva Zelandia, Estados Unidos, Canadá entre otros ${ }^{2-5}$. Estos bancos están dedicados a la nutrición del prematuro, complementando la leche de su propia madre. En Noruega la leche donada de madres con serología negativa para HIV, hepatitis, virus linfotrópico y CMV, no es pasteurizada, sólo refrigerada y congelada ${ }^{6}$.

En Chile se ha mantenido en la norma de prevención de transmisión vertical de VIH la prohibición de "lactancia en madres VIH positivo, ... la alimentación por nodrizas y por leche provenientes de bancos de leche". La norma de 2005 reconoció la inactivación del virus por pasteurización, pero señalaba la insuficiente seguridad del proceso.

La experiencia internacional y el avance en investigación respecto a los beneficios de la leche materna en prematuros de muy bajo peso de nacimiento (MBPN) permite plantear que los prematuros en Chile deben contar con el acceso a leche donada, pasteurizada y segura, como se señaló en la declaración de la Rama de Neonatologia con ocasión del V Congreso Nacional de Neonatologia en 2012.

Dos objetivos importantes de la atención neonatal se pueden lograr con la expansión del uso de LH: aumentar la proporción de LH durante la hospitalización e iniciar el estímulo enteral mínimo muy precozmente.

Efectos significativos de menor incidencia y gravedad de patologías neonatales se han documentado con el uso de LH para enterocolitis necrotizante $(\mathrm{ECN})$, sepsis, dependencia de oxígeno, retinopatía del prematuro ${ }^{7-9}$. La reducción de la gravedad de la ECN adquiere gran importancia por el compromiso del neurodesarrollo que se ha descrito en la ECN de tratamiento quirúrgico ${ }^{10}$ y los mayores costos

Recibido el 01 de julio de 2014. Última versión aceptada el 21 de julio de 2014.

Patricia Mena $(\varangle)$

Servicio de Neonatología CA Dr Sotero del Río.

Profesor Asociado Adjunto Pontificia Universidad Católica de Chile.

E-mail: mena.n.patricia@gmail.com 
de salud que presentan estos niños a largo pla$\mathrm{zo}^{11}$. A mediano plazo, estudios observacionales muestran mejor desarrollo psicomotor y mejor desarrollo conductual que es proporcional a la cantidad de leche materna recibida durante la hospitalización ${ }^{11,13}$. Un estudio controlado randomizado de fórmula versus $\mathrm{LH}$ donada, muestra evolución similar entre LH donada sin fortificar y fórmula para prematuro, pero mejor que con formula estándar ${ }^{14}$. Recientemente ha aparecido información sobre el efecto favorable de la lactancia en el desarrollo de la substancia blanca tanto en niños de pretérmino como de término ${ }^{15,16}$. Actualmente se encuentran en desarrollo tres estudios randomizados, multicéntricos, en MBPN con LH donada, adecuadamente fortificada, versus fórmula para prematuro evaluando el desarrollo cognitivo a mediano plazo ${ }^{17}$.

A más largo plazo, una de las pocas intervenciones randomizadas en prematuros, documenta un menor riesgo de enfermedades crónicas del adulto, lo que es producido por las modificaciones epigenéticas inducidas por la restricción del crecimiento, intra o extrauterino ${ }^{18}$.

Si bien la mayoría de los estudios son observacionales, los efectos observados son proporcionales a la cantidad de leche materna recibida. Un estudio aleatorizado con leche donada versus fórmula, realizado en Inglaterra a fines de los 80, demostró efectos favorables en desarrollo y en riesgo de enfermedades crónicas del adulto ${ }^{14,18}$. Estudios que aleatorizan la administración de leche donada o fórmula si la leche de su madre no es suficiente, han mostrado ventajas en morbilidad; los estudios que están en curso mostrarán las ventajas y dificultades con leche donada, de su propia madre y las fórmulas.

La LH se considera como la mejor alternativa de alimentación del niño de muy bajo peso de nacimiento (MBPN) ${ }^{19,20}$ preferentemente leche de su propia madre, pero debe ser adecuadamente fortificada con proteínas, calcio, fosforo; otros nutrientes considerados teóricamente necesarios se han agregado a los fortificadores. Idealmente, el fortificante también debiera estar basado en LH, lo que actualmente está disponible en un producto comercial de alto costo en USA, con el cual la disminución de incidencia y gravedad de la enterocolitis necrotizante es aún mayor ${ }^{21}$.

El otro aspecto importante para lo cual la leche donada es fundamental, es el riesgo de retrasar el inicio del aporte enteral. El ayuno enteral ha sido claramente documentado como un factor de riesgo de sepsis, de aumentar la duración de la oxígenoterapia, la ventilación asistida y la necesidad de nutrición parente$\mathrm{ral}^{22}$. Generalmente se retrasa el aporte enteral en un MBPN por el riesgo de enterocolitis necrotizante, práctica muy extendida, que ha ido perdiendo fundamentos. En casos de menores de 34 semanas con doppler fetal alterado, no se ha demostrado que retrasar el inicio en más de 36 a $48 \mathrm{~h}$ reduzca el riesgo de enterocolitis ${ }^{23,24}$. La tendencia actual es iniciar el aporte con LH en volumen reducido -enteral mínimo-, desde el primer día de vida, con aumento gradual lento en niños de extremo bajo peso y relativamente más rápido en niños de más de $1.000 \mathrm{~g}$. Muchas veces se indica leche materna exclusiva, y si no se cuenta con un banco de leche y un sistema activo de extracción de la leche de su madre, pueden pasar varios días sin recibir aporte enteral.

La obtención de leche materna de su propia madre para el prematuro durante su hospitalización no es fácil, ya que estas madres presentan frecuentemente complicaciones severas de salud, estrés por la condición del hijo e inmadurez mamaria de la función secretoria. La mayor dificultad es contar con leche precozmente, en la primera semana, $y$, posteriormente, mantener la producción durante el segundo mes. El volumen de leche producido por la madre del prematuro es menor a menor edad de la gestación, pero con una gran variabilidad entre las madres. Para favorecer la obtención de leche se requiere: iniciar la extracción muy precoz, en las primeras $6 \mathrm{~h}$ postparto; realizar extracción simultanea de ambos pechos; usar bombas de dos fases; utilizar masaje mamario simultáneamente al bombeo; realizar contacto piel a piel, al menos dos horas diarias; realizar succión no nutritiva al pecho, desde las 30-31 semanas, utilizar suplementadores al pecho, durante el paso de alimentación enteral a oral; apoyo del equipo de salud y de pares para el 
manejo del estrés materno; mantener vigilancia del volumen extraído para intervención educativa; y el uso de galactogogos. Todas estas actividades requieren de la disposición adecuada de personal, insumos y equipamiento apropiado.

Por los claros beneficios de la leche materna para el RNMBPN y las dificultades reales de contar con leche de su propia madre, cada vez más centros neonatales han desarrollado los bancos de leche materna. Los riesgos asociados al consumo de leche donada han sido minimizados al máximo con los exigentes protocolos de bioseguridad y trazabilidad de los bancos de leche ${ }^{5-8}$ : Estudios realizados con la inoculación de VIH en LH o con células infectadas con VIH demuestran que el proceso de la pasteurización inactiva el VIH ${ }^{25,26}$. También el CMV, potencial patógeno significativo para el prematuro, es totalmente inactivado con la pasteurización.

La pasteurización tipo Holder, es el método más utilizado y somete la leche a una temperatura constante de $62,5^{\circ} \mathrm{C}$ por $30 \mathrm{~min}$ con posterior enfriamiento rápido a $5^{\circ} \mathrm{C}$. Este método tiene la ventaja de mantener gran parte de los aspectos nutricionales de la LH y además de disminuir drásticamente o inactivar la carga bacteriológica y viral, posibilitando su uso seguro. Actualmente este método se utiliza con equipos automatizados, que aseguran los niveles de temperatura y con control bacteriológico posterior, que garantiza el proceso ${ }^{2-6}$.

Los efectos protectores de la leche materna se producen por la sinérgica acción de sus componentes nutricionales, enzimáticos, inmunológicos, antiinfecciosos, antiinflamatorios y antioxidantes. Si bien la pasteurización afecta a algunas de estas acciones (desaparecen las células, disminuye la actividad enzimática y hormonal, desaparece la actividad IgM y de complemento), sólo disminuye la actividad de IgA y lactoferrina, se conserva el aporte nutricional, la funciones de nitrógeno no proteico, la complejidad de sus ácidos grasos y de otros bioactivos $^{27}$ lo que determina sus cualidades protectoras, que las fórmulas para prematuros no logran igualar.

Estudios de costo-efectividad de la implementación de Bancos de Leche Humana indi- can que los beneficios obtenidos superan en hasta seis veces su costo ${ }^{28}$, reforzando su importancia como medida de salud pública.

La presencia de un banco de leche generalmente ha producido un impacto cultural local, con valorización de la leche materna y mejoría de los niveles de lactancia, incluyendo las madres de los RNBPN hospitalizados.

La posibilidad de contar con volúmenes de leche donada suficiente, para producir un fortificante basado en leche materna en nuestro país, es una posibilidad real, que permitiría reducir al mínimo la incidencia de ECN en el extremo bajo peso de nacimiento.

Reducir la morbilidad y secuelas, potenciando un mejor desarrollo en los prematuros de gran vulnerabilidad biológica, es un objetivo central al implementar un banco de leche. Desde el punto de vista de país, organizar una red de bancos de leche, ubicadas a lo largo del país, con un óptimo uso de los recursos físicos y humanos, y un sistema de acreditación y control del proceso, es una iniciativa pendiente de las autoridades de salud.

Conflicto de interes: La Dra. P. Mena visitó bancos de leche en Francia financiada por HSC Francia y Medela, Chile.

\section{Referencias}

1.- Red Brasileña de bancos de leche: http://www.redeblh. fiocruz.br/

2.- Human Milk Banking Association of North America: Guidelines for the establishment and operation of a donor human milk bank. Forth Worth, TX: Human Milk Banking Association of North America; 2011. http:// www.hmbana.org

3.- Hartmann BT, Pang WW, Keil AD, Hartmann PE, Simmer $K$ : Best practice guidelines for the operation of a donor human milk bank in an Australian NICU. Early Hum Develop 2007; 83: 667-73.

4.- National Institute for Health and Clinical Excellence (2010) Donor breast milk banks: the operation of donor breast milk bank services. London: National Institute for Health and Clinical Excellence. Available from: www. nice.org.uk/guidance/CG93

5.- Ministere de la Santé et des Sports. Missions, autorisation et conditions techniques d'organisation et de 
fonctionnement des lactariums Décret no 2010-805 du 13 juillet 2010. http://sdp.perinat-france.org/ADLF/ textes_legislation_adlf.php

6.- Grøvslien AH, Grønn M: Donor milk banking and breastfeeding in Norway. J Hum Lact 2009; 25: 206-10.

7.- McGuire W, Anthony MY: Donor human milk versus formula for preventing necrotizing enterocolitis in preterm infants: systematic review. Arch Dis Child Fetal Neonatal Ed. 2003; 88: F11-F14.

8.- Schanler RJ, Lau C, Hurst NM, Smith EO: Randomized trial of donor human milk versus preterm formula as substitutes for mothers' own milk in the feeding of extremely premature infants. Pediatrics 2005; 116: 400-6.

9.- Patel AL, Johnson TJ, Engstrom JL, et al: Impact of early human milk on sepsis and health-care costs in very low birth weight infants. J Perinatol 2013; 33: 514-9.

10.- Hintz SR, Kendrick DE, Stoll et al: NICHD Neonatal Research Network: Neurodevelopmental and growth outcomes of extremely low birth weight infants after necrotizing enterocolitis. Pediatrics 2005; 115: 696-703.

11.- Ganapathy V, Hay J, Kim J, Lee M, Rechtman D: Long term healthcare costs of infants who survived neonatal necrotizing enterocolitis: a retrospective longitudinal study among infants enrolled in Texas Medicaid. BMC Pediatrics 2013; 13: 127.

12.- Vohr BR, Poindexter BB, Dusick AM, McKinley LT: NICHD Neonatal Research Network: Persistent beneficial effects of breast milk ingested in the neonatal intensive care unit on outcomes of extremely low birth weight infants at 30 months of age. Pediatrics 2007; 120: e953-9.

13.- Horwood LJ, Darlow BA, Mogridge N: Breast milk feeding and cognitive ability at 7-8 years. Arch Dis Child Fetal Neonatal Ed 2001; 84: F23-7.

14.- Lucas A, Morley R, Cole TJ, et al: Early diet in preterm babies and developmental status in infancy. Arch Dis Child 1989; 64: 1570-8.

15.- Pogribna U, Yu X, Burson K, et al: Perinatal Clinical Antecedents of White Matter Microstructural Abnormalities on Diffusion Tensor Imaging in Extremely Preterm Infants. www.plosone.org 2013; 8 (8): e72974.

16.- Herba C, Roza S, Govaert P, et al: Breastfeeding and early brain development: the Generation R study. Ma- ternal and Child Nutrition 2013; 9: 332-49.

17.- Colaizy T: Donor Human Milk for Preterm Infants What It Is, What It Can Do, and What Still Needs to Be Learned Clin Perinatol 2014; 41: 437-50.

18.- Singhal A, Fewtrell $M$, Cole TJ, et al: Low nutrient intake and early growth for later insulin resistance in adolescents born preterm. Lancet 2003; 361: 1089-97.

19.- Section on Breastfeeding: Breastfeeding and the use of human milk. Pediatrics 2012; 129: e827-41.

20.- Arslanoglu $S$, Corpeleijn W, Moro G, et al: ESPGHAN. Donor human milk for preterm infants: current evidence and research directions. J Pediatr Gastroenterol Nutr 2013; 57: 535-42.

21.- Sullivan S, Schanler RJ, Kim JH, et al: An exclusively human milk-based diet is associated with a lower rate of necrotizing enterocolitis than a diet of human milk and bovine milk-based products. J Pediatr 2010; 156: 562-7.

22.- Wilson DC, Cairns P, Halliday HL, et al: Randomised controlled trial of an aggressive nutritional regimen in sick very low birth weight infants. Arch Dis Child 1997; 77: F4-11.

23.- Kempley S, Gupta N, Linsell L, Dorling J, et al: Feeding infants below 29 weeks' gestation with abnormal antenatal Doppler: analysis from a randomised trial. Arch Dis Child Fetal Neonatal Ed 2014; 99: F6-11.

24.- Leaf A, Dorling J, Kempley S, et al: Early or delayed enteral feeding for preterm growth-restricted infants: a randomized trial. Pediatrics 2012; 129: e1260-8.

25.- Eglin RP, Wilkinson AR: HIV infection and pasteurisation of breast milk. Lancet 1987; 1 (8541): 1093.

26.- Terpstra FG, Rechtman DJ, Lee ML, et al: Antimicrobial and antiviral effect of high-temperature short-time (HTST) pasteurization applied to human milk. Breastfeed Med 2007; 2: 27-33.

27.- Ewaschuk JB, Unger S, Harvey S, O'Connor DL, Field $C J$ : Effect of pasteurization on immune components of milk: implications for feeding preterm infants. Appl Physiol Nutr Metab 2011; 36: 175-82.

28.- Arnold LD: The cost-effectiveness of using banked donor milk in the neonatal intensive care unit: prevention of necrotizing enterocolitis. J Hum Lact 2002; 18: 1727. 